\title{
Exome Sequencing Identifies a Novel SIN3A Variant in a Patient with Witteveen-Kolk Syndrome
}

\author{
Monica Penon-Portmann ${ }^{\mathrm{a}, \mathrm{b}}$ Colleen M. Carlston ${ }^{\mathrm{a}}$ Pierre-Marie Martin ${ }^{\mathrm{b}}$ \\ Anne Slavotinek ${ }^{a, b}$ \\ aDivision of Medical Genetics, Department of Pediatrics, University of California San Francisco, San Francisco, CA, USA; \\ bInstitute for Human Genetics, University of California San Francisco, San Francisco, CA, USA
}

\section{Established Facts}

- Witteveen-Kolk syndrome (WITKOS) is a rare neurodevelopmental syndrome characterized by mild intellectual disability and a recognizable facial gestalt.

- WITKOS is caused by heterozygous loss-of-function variants in SIN3A.

\section{Novel Insights}

- Here, we report the first patient with Hispanic ancestry affected by WITKOS with a novel truncating variant in the SIN3A gene.

- This patient supports the currently described phenotype for WITKOS in more diverse populations.

\section{Keywords}

$15 q 24$ deletion $\cdot$ Developmental delay · Diverse populations · SIN3A - Witteveen-Kolk syndrome

\begin{abstract}
Witteveen-Kolk syndrome (WITKOS; OMIM \#613406) is a recently described, rare neurodevelopmental syndrome characterized by mild intellectual disability and a recognizable facial gestalt. WITKOS is caused by heterozygous loss-offunction variants in $\operatorname{SIN} 3 A$. It shares some features with $15 q 24$ deletion syndrome but to date has only been described in a limited number of patients mostly of Northern
\end{abstract}

European ancestry. Here, we report the first patient with Hispanic ancestry to our knowledge diagnosed with WITKOS, who has a novel, truncating variant in the SIN3A gene. Clinical exome sequencing performed in-house using a custom bioinformatics pipeline identified a de novo heterozygous, nonsense variant in SIN3A, c.1015C>T (p.GIn339Ter) that has not been previously described in the literature. This 3-yearold boy with WITKOS demonstrated classic features including mild developmental delay and triangular facies with hypotelorism and deep-set, hooded eyes. This patient supports the currently described phenotype for WITKOS in more diverse populations.

(c) 2022 The Author(s)

Published by S. Karger AG, Basel
(C) 2022 The Author(s)

Published by S. Karger AG, Basel

This is an Open Access article licensed under the Creative Common Attribution-NonCommercial-4.0 International License (CC BY-NC) (http://www.karger.com/Services/OpenAccessLicense), applicable to the online version of the article only. Usage and distribution for commercial purposes requires written permission. 


\section{Introduction}

Witteveen-Kolk syndrome (WITKOS; OMIM \#613406) is a rare neurodevelopmental syndrome characterized by mild intellectual disability and a recognizable facial gestalt. WITKOS is caused by switch-insensitive 3 family member A (SIN3A) haploinsufficiency which is thought to lead to aberrant cortical neurogenesis, as demonstrated by the decrease in cortical progenitors observed in the $\operatorname{Sin} 3 a$ mouse model [Witteveen et al., 2016]. The causal role of haploinsufficiency for SIN $3 A$ is supported by the significant clinical overlap between the phenotypes of WITKOS and of patients with de novo, atypical chromosome 15q24 deletion syndrome [Mefford et al., 2012; Witteveen et al., 2016; Narumi-Kishimoto et al., 2019; Balasubramanian et al., 2021].

SIN3A encodes an epigenetic regulator involved in intellectual disability and autism spectrum disorders [Hassig et al., 1997; Laherty et al., 1997; Chaubal and Pile, 2018; Narumi-Kishimoto et al., 2019]. SIN3A interacts with $\mathrm{MeCP} 2$, the protein responsible for Rett syndrome, a clinically distinct, well-studied neurodevelopmental condition classically observed in girls [Witteveen et al., 2016; Ip et al., 2018].

To date, less than 50 individuals with WITKOS have been published in the medical literature [Witteveen et al., 2016; Ferrer et al., 2019; Narumi-Kishimoto et al., 2019; Balasubramanian et al., 2021; Ercoskun and Yuce Kahraman, 2021]. Herein, we a report the first patient with Hispanic-Caucasian ancestry affected by WITKOS with a novel truncating variant in the SIN $3 A$ gene.

\section{Case Report}

The patient is a 3-year-old male who was born at 31 weeks and 6 days via spontaneous vaginal delivery after premature rupture of membranes and preterm labor. The pregnancy was complicated by oligohydramnios in the 3rd trimester and a shortened cervix at 21 weeks managed with cerclage. Steroid, magnesium sulfate, and antibiotics were administered prior to delivery. His mother had a history of deep venous thrombosis treated with heparin and enoxaparin during pregnancy. He was small for gestational age, with a weight of 1,250 $\mathrm{g}$ (9th centile), length $41 \mathrm{~cm}$ (38th centile), and head circumference $27 \mathrm{~cm}$ (6.5th centile), plotted in Fenton preterm boy curves. Prenatal ultrasounds prior to 32 weeks were reportedly normal. He had Apgar scores of 8 and 9. He was the first child born to a 38-year-old, G1P1 mother of Hispanic ancestry and a 56-year-old father of Caucasian ancestry with healthy 11-yearold fraternal twin half-siblings. There is no family history of developmental delays.

Placental studies revealed a singleton placenta $190 \mathrm{~g}$ (5th centile) with acute chorioamnionitis and eccentric cord insertion. In- fectious workup was unrevealing with negative prenatal group B Streptococcus culture, prenatal maternal TORCH serologies, and urine test for cytomegalovirus. Initial head ultrasound at day 7 of life showed echogenicity of the occipital bilateral lateral ventricles and mild ventricular dilation suggestive of a recent intraventricular hemorrhage. Serial head ultrasounds showed still prominent bilateral ventricles but with no evidence of bleeding. Brain magnetic resonance imaging (MRI) at 5 weeks of age documented mild ventriculomegaly, tiny amounts of blood product layering within the occipital horns bilaterally, and adequate myelination for age with no migration anomalies or congenital/structural abnormalities identified. He had retinopathy of prematurity with an eye exam at 1 month of age that showed immature vascularization of zone 3 bilaterally. Hearing screen and newborn screen were unremarkable.

He was referred to the genetics clinic for evaluation due to global developmental delay, hypotonia, plagiocephaly, and congenital torticollis. At 6 months of age (adjusted to age 4 months for prematurity) he did not have full head control and was not bringing his hands together to the midline, but he was able to roll and maintain good eye contact. He had truncal hypotonia and slight appendicular hypertonia that was mostly present in the upper extremities.

At 2 years and 6 months of age he remained delayed but with progress in all 4 areas of development. He was pulling to stand, cruising, but not yet walking independently. He could stack 7-8 blocks and had a pincer grasp but was not yet copying a line. $\mathrm{He}$ had a few words and had reportedly good eye contact with parents and liked playing with other children. There was no history of seizures, developmental regression, or behavioral abnormalities. $\mathrm{He}$ has also had a history of periorificial and atopic dermatitis and his father endorsed similar dermatologic issues in his own childhood.

On first evaluation at 2 years and 6 months of age, growth parameters were weight $11 \mathrm{~kg}$ ( 2 nd centile), height $87 \mathrm{~cm}$ (12th centile), and head circumference $48.5 \mathrm{~cm}$ (26th centile), plotted in CDC growth curves for males 2-20 years and Nellhaus Boys for head circumference. He had closely spaced, deep-set eyes that were mildly hooded, mild bilateral ptosis, and downslanting palpebral fissures (Fig. 1). He had a broad and prominent forehead and a slightly triangular face with proportionately large ears. He had brachydactyly and 5th finger clinodactyly. He had slight hypotonia. There were no ectodermal findings or joint hypermobility.

Investigations prior to exome sequencing included a single nucleotide polymorphism (SNP) array performed on Illumina $\mathrm{Cy}$ toSNP-850 K platform (genome build hg19) from peripheral blood that was normal $(\operatorname{arr}(1-22) \times 2,(\mathrm{X}, \mathrm{Y}) \times 1)$. Fragile $\mathrm{X}$ testing showed a normal FMR 1 allele with 29 repeats. Whole exome sequencing (WES) was performed as a trio (proband with both biological parents) as part of the Prenatal and Pediatric Genomic Sequencing Program (P3EGS) at the University of California, San Francisco (UCSF). Written informed consent was obtained from legal guardians for WES, publication of this report, and accompanying images. WES was performed as a clinical test using a bioinformatics pipeline developed by the Institute for Human Genetics (IHG) at UCSF as published previously [Mendelsohn et al., 2020]. Findings were evaluated using the American College of Medical Genetics and Genomics (ACMG) criteria for variant calling [Richards et al., 2015]. The results of the exome trio showed a heterozygous de novo truncating variant in SIN3A, NM_015477.2: c.1015C>T (p.Gln339Ter) (genome build hg19). 


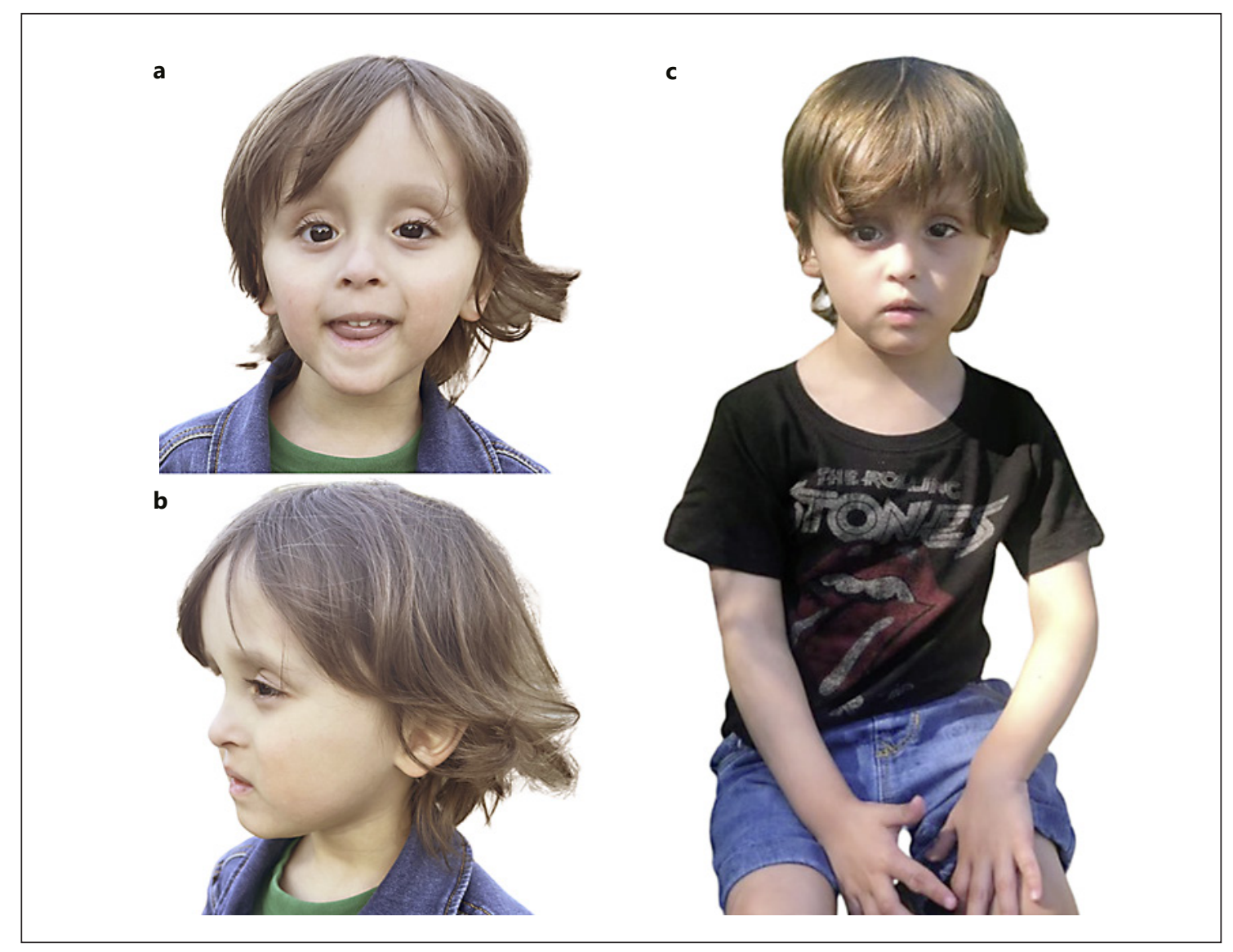

Fig. 1. Clinical photographs of our patient at 3 years of age. a, b Broad and prominent forehead and a slightly triangular face with proportionately large ears. Closely spaced, deep-set eyes that are mildly hooded, mild bilateral ptosis, and downslanting palpebral fissures. c Fifth finger clinodactyly and brachydactyly.

\section{Discussion}

We describe a 3-year-old male with WITKOS with a novel truncating variant in SIN3A. To our knowledge this is the first case of WITKOS in a patient of Hispanic ancestry. WITKOS is an autosomal dominant syndrome caused by loss-of-function variation in SIN3A, a highly conserved gene intolerant to both missense and loss-offunction variation with $\mathrm{pLI}=1$ and a missense $\mathrm{Z}$-score = 4.39 [Karczewski et al., 2020].

This variant we report here was considered pathogenic per ACMG criteria [Richards et al., 2015]. It is a truncating variant in a gene where loss-of-function is a known mechanism of disease (PVS1). It is also a de novo variant in an affected patient with no family history of similar developmental delays (PS2) and it is absent from controls in gnomAD (PM2) [Richards et al., 2015; Karczewski et al., 2020]. The variant is predicted to cause a premature stop codon in exon 7 of 21 (Fig. 2) and the transcript is predicted to be susceptible to nonsense-mediated decay (NMD), consistent with haploinsufficiency as the mechanism of disease. Even if a protein product were produced from transcripts that escape NMD, the variant would truncate the highly conserved paired amphipathic helix domain 2 (PAH2), which plays a critical role in the interaction between core components of the Sin 3 scaffold complex [Chaubal and Pile, 2018]. Loss-of-function variants downstream to the variant shown in this case at residue 339 (p.Gln339Ter) have been described in other cases of WITKOS (Fig. 3). Predominantly truncating variants have been ascribed to individuals with WITKOS, yet missense and splice site variants have also been described. Missense variants were predicted to be likely pathogenic given they occurred de novo, were located in a critical functional domain, were absent from controls, and showed deleterious predictions in silico [Balasubramanian et al., 2021]. 


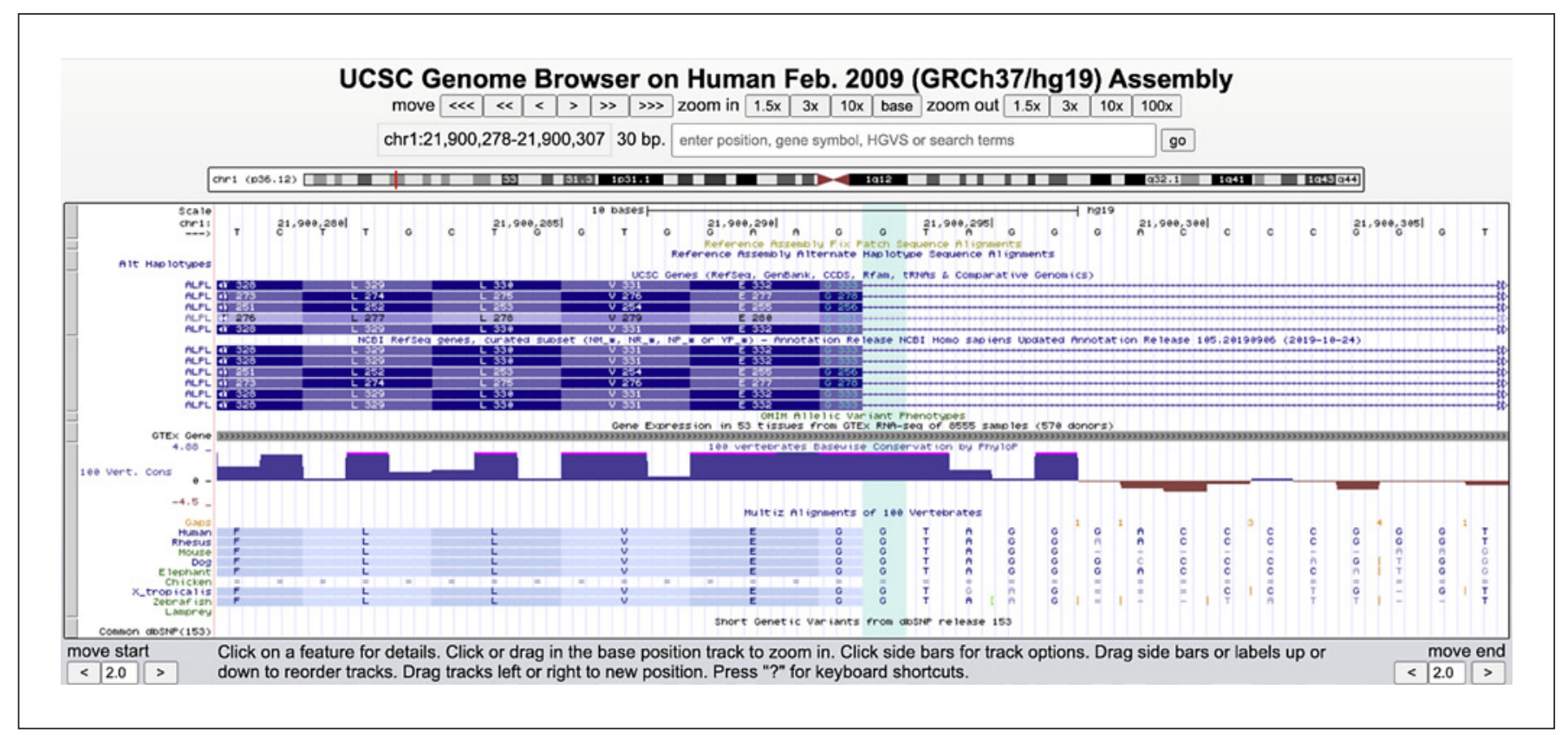

Fig. 2. View from UCSC Genome Browser of the de novo variant highlighted in light-blue in SIN3A: NM_015477.2, c.1015C > T (p.Q339Ter). Multiz Alignments of 100 Vertebrates shows this residue is highly conserved. Region shown: chr1:21,900,278-21,900,307 (genome build GRCh37).

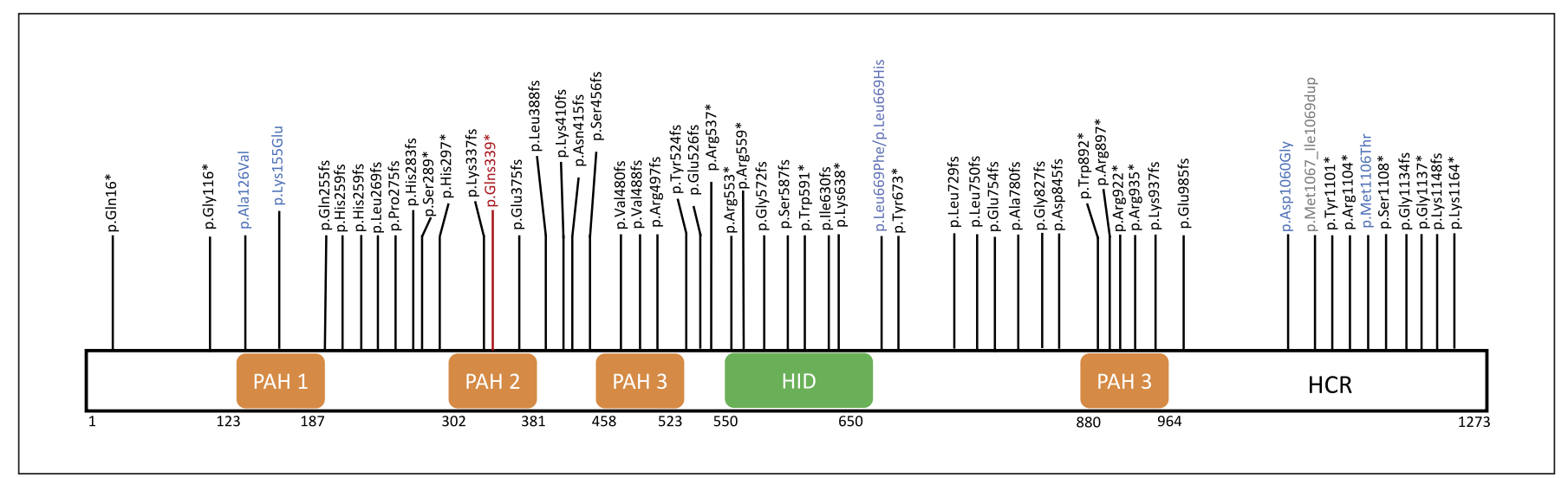

Fig. 3. SIN $3 A$ protein structure and identified SIN3A pathogenic $(P)$ and likely pathogenic (LP) variants in patients with WITKOS. Variants were compiled from the literature and ClinVar catalogued P and LP variants. The pathogenic variant described in this patient is in maroon. Missense variants are indicated in blue, loss-of-function variants indicated in black (frameshift and nonsense variants) and a duplication is indicated in grey. PAH, paired amphipatic helix domain; HID, histone deacetylase interacting domain; HCR, highly conserved region.

$S I N 3 A$ is an important regulator of mammalian cerebral cortex development. Sin $3 \mathrm{a}$ is highly expressed in the cortex and Sin $3 a$ in vivo knockdowns lead to cortical progenitor decrease, suggesting it plays an essential role during neurogenesis [Witteveen et al., 2016]. There is a variable educational attainment in patients with WITKOS and impairment ranges from mild to severe [Ferrer et al.,
2019; Narumi-Kishimoto et al., 2019; van Dongen et al., 2020; Ercoskun and Yuce Kahraman, 2021]. A recent series that also evaluated behavior demonstrated differences in attention and social interactions that to date have not been noted in our patient [van Dongen et al., 2020].

Our analysis of the clinical features presented in cases described to date suggest that the most common findings 
Table 1. Phenotypic compilation of our patient and previously reported individuals with truncating variants in SIN3A

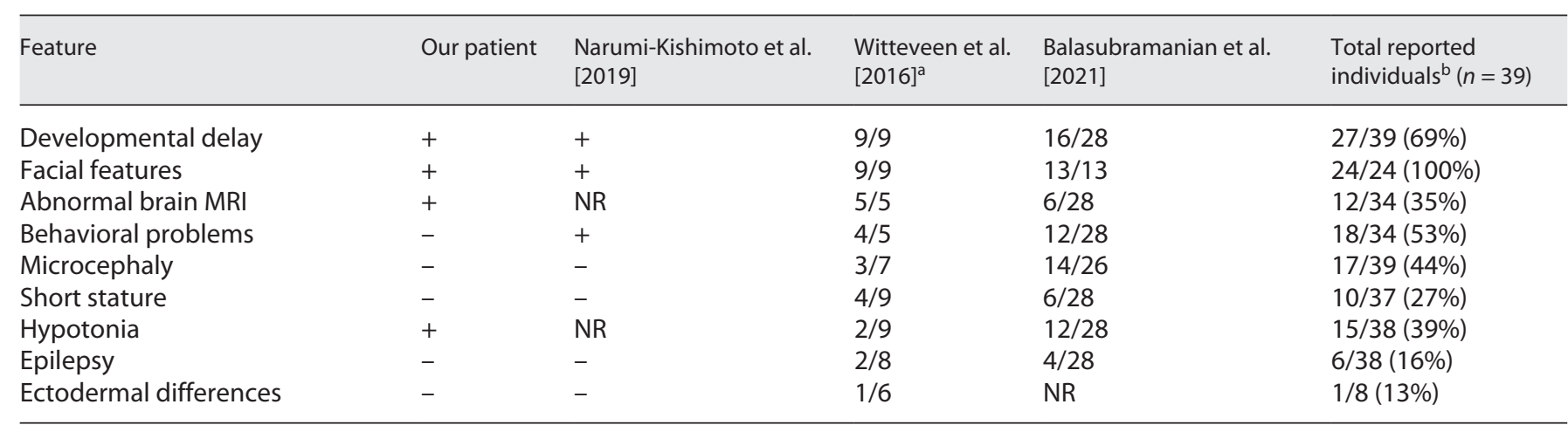

${ }^{a}$ Nine cases were reported by Witteveen et al. [2016] with loss-of-function variants in SIN3A. As not all features were reported in all patients, the denominator represents cases with positive or negative reported findings. ${ }^{b}$ Patients reported by Witteveen et al. [2016], NarumiKishimoto et al. [2019]. NR, not reported.

of WITKOS are developmental differences, which can be mild as found in this child, characteristic non-familial features, and behavioral differences in agreement with findings from Balasubramanian et al. [2021] (Table 1). To our knowledge, intrauterine growth restriction and polyhydramnios have not been described in other patients with WITKOS. Further studies are required to ascertain a potential clinical correlation in this disorder. Initial recommendations for evaluation based on findings in WITKOS patients should include a developmental assessment, brain imaging, evaluation by ophthalmology and audiology, and continued surveillance for developmental delays or new-onset seizures. Clinically there were no significant differences in the presentation of this patient compared to previously reported non-Hispanic patients, but photographs of patients from diverse backgrounds may help clinicians to better recognize rare conditions in these populations.

In summary, we present a novel SIN3A truncating variant in a male with WITKOS characterized by features including mild developmental delays, reduced weight, and triangular facies with protuberant ears and deep-set, hooded eyes. His features support the currently recognized phenotypic spectrum of this emerging condition.

\section{Acknowledgement}

The authors are grateful to the family for their willingness to share this report with the medical community. We would also like to thank the clinical laboratory at UCSF.

\section{Statement of Ethics}

All procedures, including the informed consent process, were conducted in accordance with the ethical standards of the responsible committee on human experimentation (institutional and national) and with the Helsinki Declaration. Written informed consent was obtained from the parent/legal guardian of the patient for publication of the details of their medical case and any accompanying images.

\section{Conflict of Interest Statement}

The authors declare no conflict of interest.

\section{Funding Sources}

The authors have no funding sources to declare.

\section{Data Availability Statement}

All data generated or analyzed during this study are included in this article. Further inquiries can be directed to the corresponding author.

\section{Author Contribution}

All authors read and approved the manuscript. A.S. designed, coordinated the study, and performed clinical investigations. M.P.P. and C.C. drafted the manuscript. P.M.M. performed molecular analysis. A.S. and P.M.M. provided critical revisions. 


\section{References}

Balasubramanian M, Dingemans AJM, Albaba S, Richardson R, Yates TM, Cox H, et al. Comprehensive study of 28 individuals with SIN3A-related disorder underscoring the associated mild cognitive and distinctive facial phenotype. Eur J Hum Genet. 2021;29(4): 625-36.

Chaubal A, Pile LA. Same agent, different messages: Insight into transcriptional regulation by SIN3 isoforms. Epigenetics Chromatin. 2018;11:17-1.

van Dongen LCM, Wingbermühle E, Dingemans AJM, Bos-Roubos AG, Vermeulen K, PopPurceleanu M, et al. Behavior and cognitive functioning in Witteveen-Kolk syndrome. Am J Med Genet A. 2020;182:2384-90.

Ercoskun P, Yuce Kahraman C. Witteveen-Kolk syndrome : The first patient from Turkey. Am J Med Genet Part A. 2021;185:617-9.

Ferrer A, Schultz-Rogers L, Kaiwar C, Kemppainen JL, Klee EW, Gavrilova RH. Three Rare Disease Diagnoses in One Patient through Exome Sequencing. Cold Spring Harb Mol Case Stud. 2019;5(6):a004390.
Hassig CA, Fleischer TC, Billin AN, Schreiber SL, Ayer DE. Histone deacetylase activity is required for full transcriptional repression by mSin3A. Cell. 1997;89:341-7.

Ip JPK, Mellios N, Sur M. Rett syndrome: Insights into genetic, molecular and circuit mechanisms. Nat Rev Neurosci. 2018;19:368-82.

Karczewski KJ, Francioli LC, Tiao G, Cummings BB, Alföldi J, Wang Q, et al. The mutational constraint spectrum quantified from variation in 141,456 humans. Nature. 2020;581: 434-44.

Laherty CD, Yang WM, Sun JM, Davie JR, Seto E, Eisenman RN. Histone deacetylases associated with the $\mathrm{mSin} 3$ corepressor mediate Mad transcriptional repression. Cell. 1997;89:34956.

Mefford HC, Rosenfeld JA, Shur N, Slavotinek AM, Cox VA, Hennekam RC, et al. Further clinical and molecular delineation of the 15q24 microdeletion syndrome. J Med Genet. 2012;49:110-8.
Mendelsohn BA, Beleford DT, Abu-El-Haija A, Alsaleh NS, Rahbeeni Z, Martin PM, et al. A novel truncating variant in ring finger protein 113A (RNF113A) confirms the association of this gene with X-linked trichothiodystrophy. Am J Med Genet Part A. 2020;182:513-20.

Narumi-Kishimoto Y, Araki N, Migita O, Kawai T, Okamura K, Nakabayashi K, et al. Novel SIN3A mutation identified in a Japanese patient with Witteveen-Kolk syndrome. Eur J Med Genet. 2019;62:103547.

Richards S, Aziz N, Bale S, Bick D, Das S, GastierFoster J, et al. Standards and guidelines for the interpretation of sequence variants: A joint consensus recommendation of the American College of Medical Genetics and Genomics and the Association for Molecular Pathology. Genet Med. 2015;17:405-24.

Witteveen JS, Willemsen MH, Dombroski TC, Van Bakel NH, Nillesen WM, Van Hulten JA, et al. Haploinsufficiency of MeCP2-interacting transcriptional co-repressor SIN3A causes mild intellectual disability by affecting the development of cortical integrity. Nat Genet. 2016;48:877-87. 